\title{
Implantoplasty in the surgical treatment of peri-implantitis: a case series
}

Paolo MORANDI*, Francesco AZZOLA*, Nicolò CAVALLI* , Clotilde AUSTONI*, Bruno BARBARO*, Luca FRANCETTI*

*Università degli Studi di Milano, Department of Biomedical, Surgical and Dental Sciences dir. Roberto L. Weinstein. IRCCS Istituto Ortopedico Galeazzi, Milan, Italy

\section{Abstract}

Most of the time non-surgical treatment of perimplantitis appears to be insufficient and a surgical intervention is necessary. The primary purpose is to have access for the debridement and decontamination of the implant surface: bacterial biofilm and calcified deposits are removed favoring healing and reducing the risk of pathology progression. The flap design should preserve the soft tissues as much as possible, for example facilitating the removal of the contaminated tissue collar from the implant's neck. Decontamination of the exposed implant by air-abrasive systems, laser or implantoplasty can be considered as auxiliary methods for regenerative or resective surgery; they may allow you to obtain better results than conventional treatment alone but the clinical benefits and the evidence reported in Literature are limited. Implantoplasty usually consists of removing the implant threads and smoothening rough implant surfaces with rotary instruments: the purpose is firstly to polish the implant surface, thereby removing the entire outmost infected layer of titanium and creating a new sterile surface structure and secondly to render the affected implant surface less plaque-retentive by reducing the surface roughness.
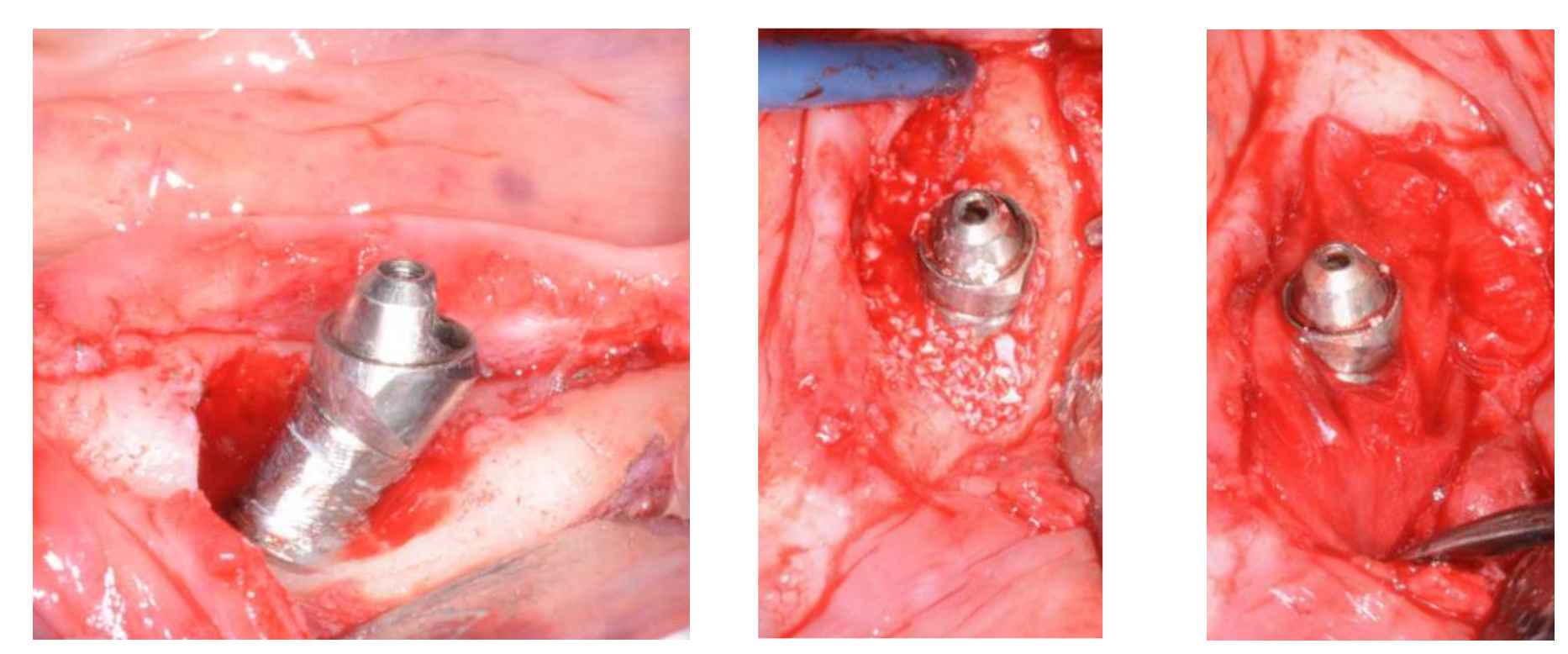

Depending on the different clinical cases, it is possible to opt for a resective or regenerative approach: in areas of poor aesthetic relevance, resective surgery and apical repositioning of the flaps can be used to reduce the probing pocket depth and improve the access for oral hygiene. On the contrary, in the aesthetic areas the exposure of titanium surfaces represents a unpleasant event to the patient that limits the use of resective therapy.

\section{Background and Aim}

Background: Peri-implantitis is a biofilm-related disease characterized by tissues inflammation and bone resorption. Despite the absence of accepted protocols, different therapeutic approaches were proposed as non-surgical therapy, access flap, resective and regenerative surgery associated to various decontamination methods of the implant surface. A beneficial effect was nevertheless reported as a result of implantoplasty in association with a resective or regenerative surgical approach.
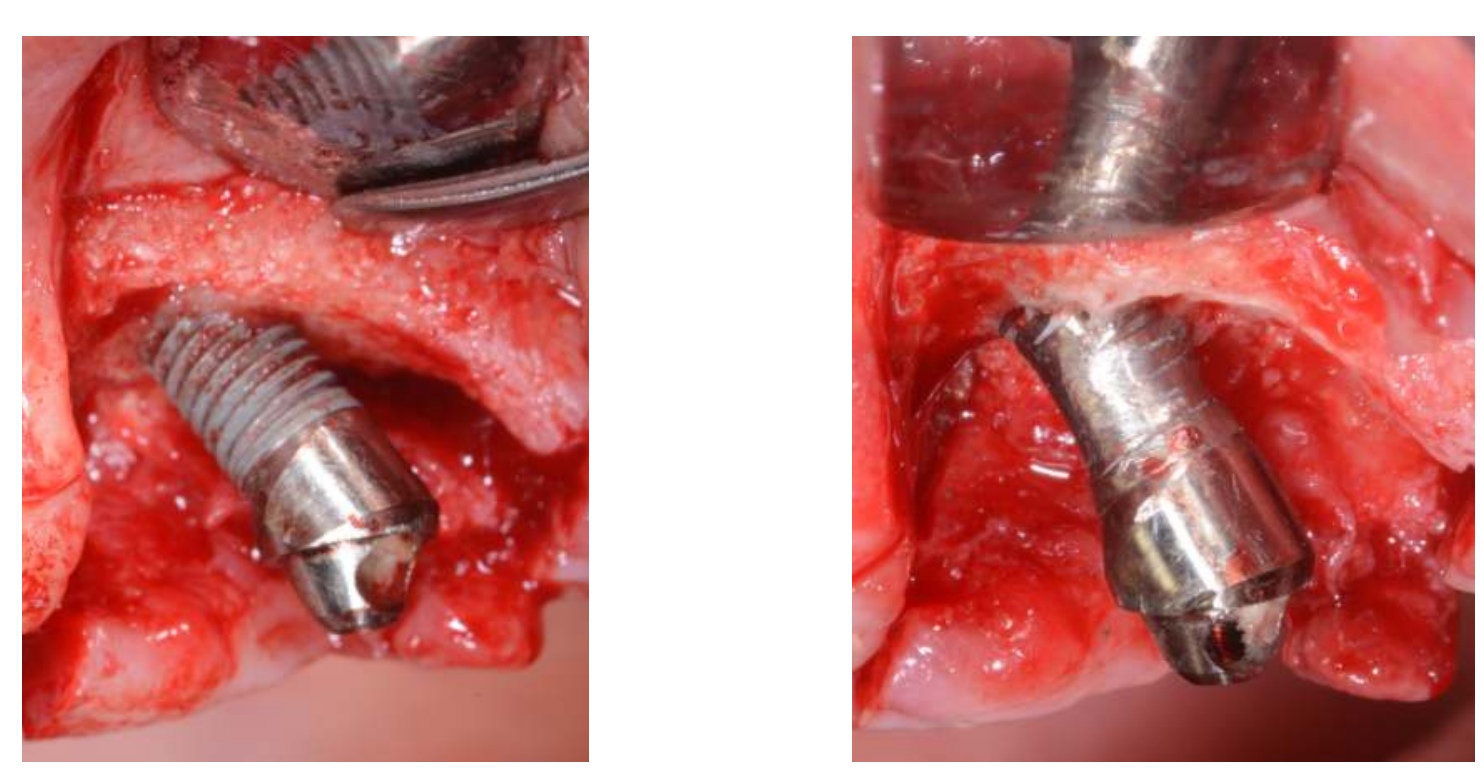

Aim/Hypothesis: The aim of this study was to observe the clinical effects of implantoplasty applied in different surgical approaches as resective surgery and regenerative surgery.

\section{Methods and Materials}

Fifteen patients with peri-implantitis were enrolled during a period of 30 months; the overall number of implants involved was 34 . All patients were months; the overall number of implants involved was 34 . All patients were
initially treated with non-surgical therapy in order to decrease peri-implant tissues inflammation and 6-8 weeks later the surgical treatment was performed. The surgical approach was chosen based on the anatomy of the bone defect. In bone defects with limited infra-bone portion $(\leq 4 \mathrm{~mm})$ and where the peri-implant ostectomy was an acceptable biologic cost for adjacent teeth or implants a resective surgical approach was chosen and implantoplasty was performed on the resulting exposed implant surface. In deep infra bone defects $(>4 \mathrm{~mm})$ and in those defects where the resection of bone wouldn't be acceptable a regenerative surgical approach was chosen. Six months after surgery all patients were reevaluated and probing depth, bone level and peri-implant soft tissues inflammation parameters (redness, bleeding-on-probing, swelling) were recorded.

\section{Results}

Two implants in two different patients treated with a combination of regenerative surgery and implantoplasty failed due to a lack a primary closure after surgery: one was removed 4 months after surgery, the other implant needed a surgical re-entry three weeks and was then dropped out. Six months after surgery all the other implants showed no signs of peri-implant inflammation, probing depths $\leq 5 \mathrm{~mm}$ and no further radiographic bone resorptions; mean probing depth (PD) gain was 2,27 $\mathrm{mm}$. No negative side effects linked to the dispersion of metal filing were recorded.

\section{PD reduction $(\mathrm{mm})$}

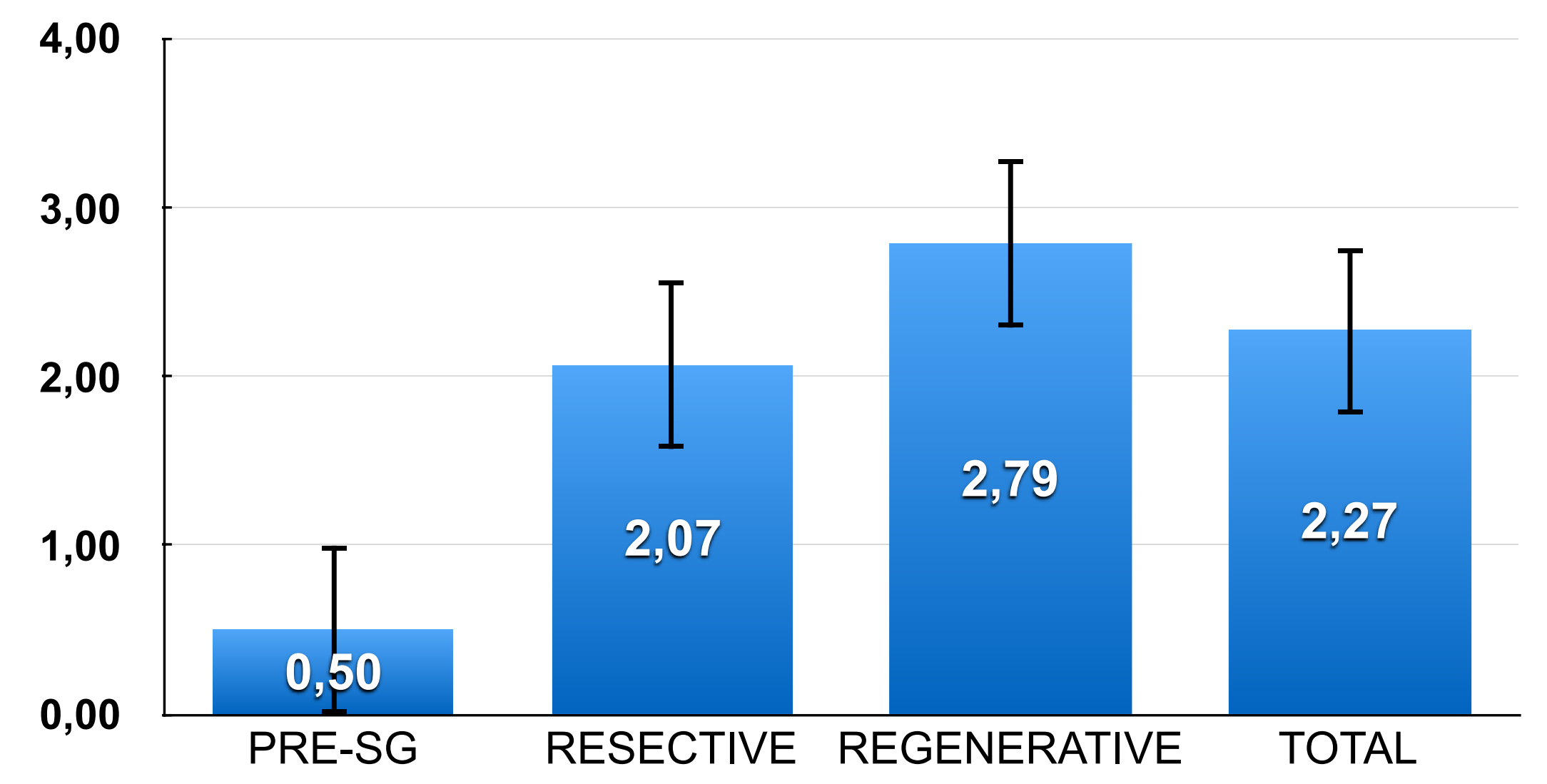

\section{Conclusions}

The association of implantoplasty and a surgical treatment of periimplantitis resulted in an improvement of all clinical parameters regarding the peri-implant tissues and could therefore be considered a safe and effective treatment option.
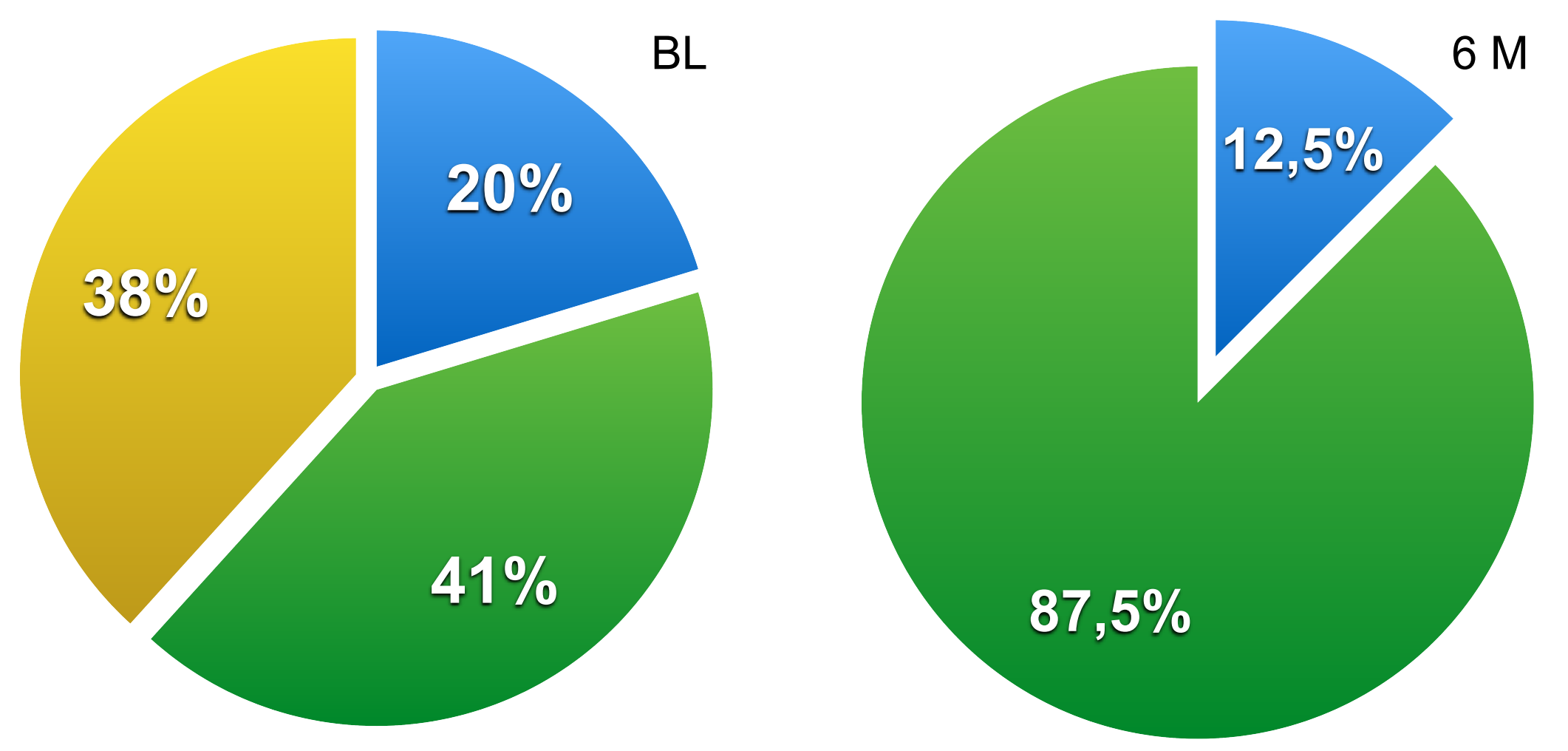

$4 \mathrm{~mm}<\mathrm{PD} \leq 5 \mathrm{~mm} \bigcirc 0 \mathrm{~mm} \leq \mathrm{PD} \leq 4 \mathrm{~mm} \bigcirc \mathrm{PD}>5 \mathrm{~mm}$

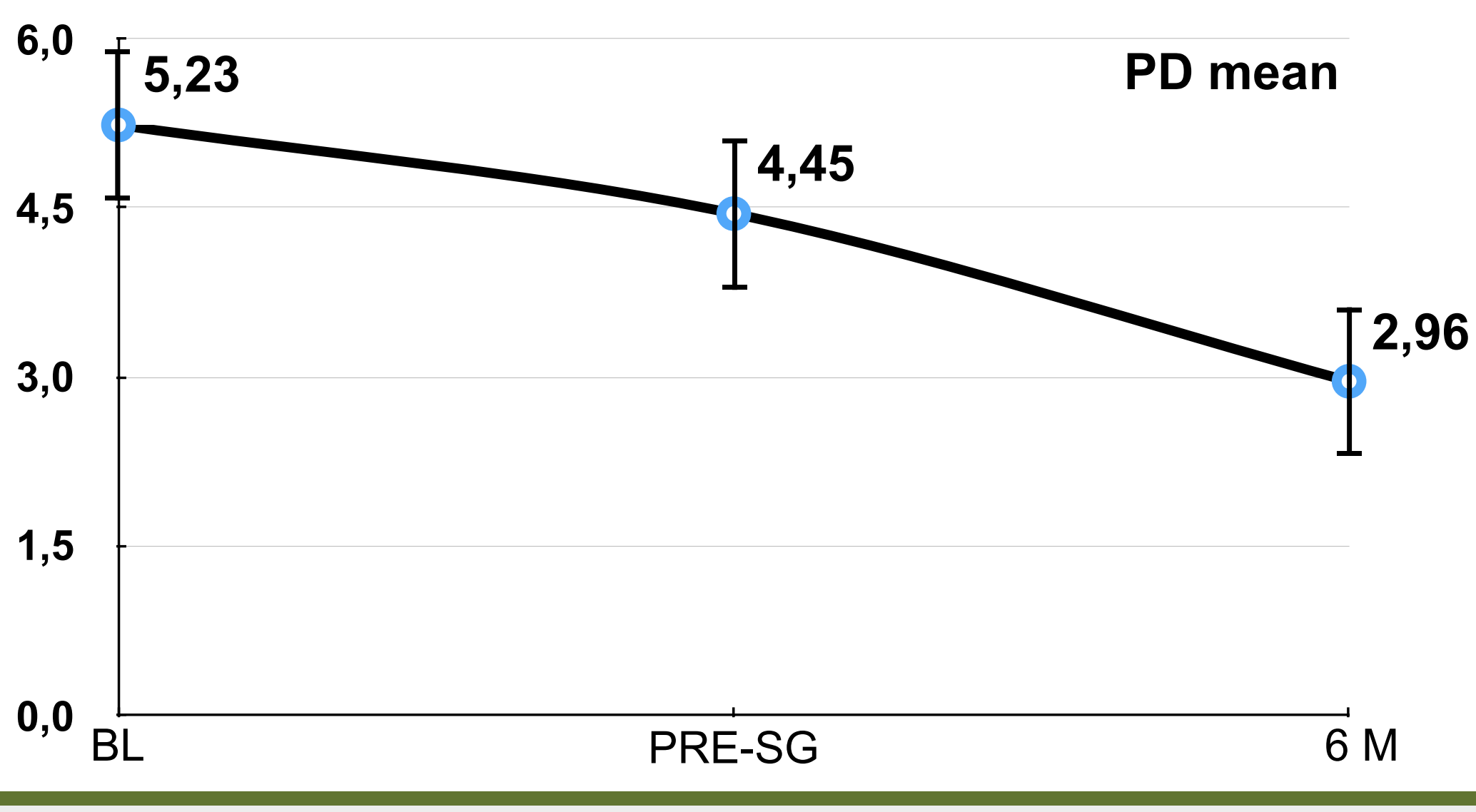

References

1. Therapy of peri-implantitis with resective surgery. A 3-year clinical tria on rough screw-shaped oral implants. Part l: clinical outcome, Clin Oral Implants Res, 2005; 16: 9-18.

2. Periimplantitis Treatment: Long-Term Comparison of Laser Decontamination and Implantoplasty Surgery, Implant Dentistry, 2016; 25(5): 646-649.

3. Combined surgical therapy of advanced peri- implantitis evaluating two methods of surface decontamination: a 7-year follow-up observation, Clin Periodontol 2017; 44: 337-342. 